\title{
Role of Clostridium perfringens Toxins in Necrotic Enteritis in Poultry
}

\author{
M Flores-Díaz, E Barquero-Calvo, M Ramírez, and A Alape-Girón
}

\section{Contents}

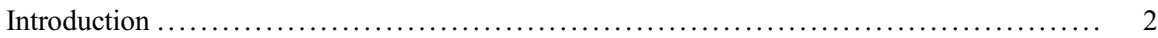

Necrotic Enteritis: Symptoms, Pathological Findings, and Diagnosis ................. 3

Clostridium perfringens Toxins Involved in Necrotic Enteritis ...................... 5

C. perfringens Phospholipase C (Cp-PLC) .................................... 5

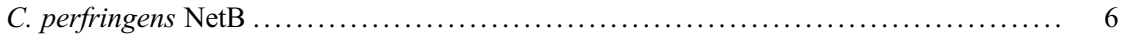

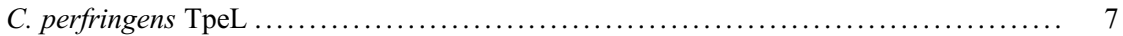

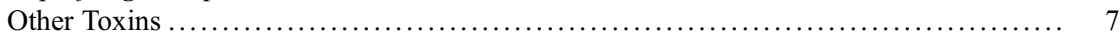

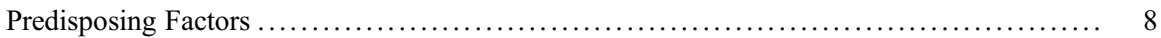

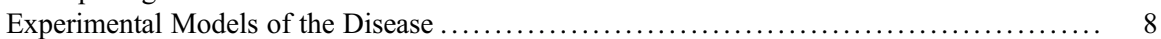

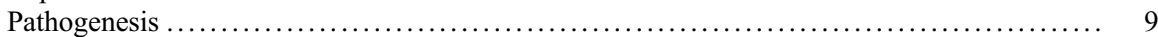

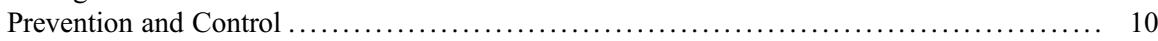

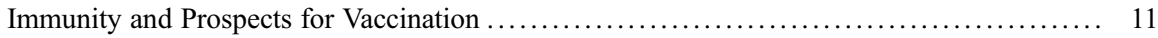

M. Flores-Díaz $(\triangle)$

Instituto Clodomiro Picado, Facultad de Microbiología, Universidad de Costa Rica, San José, Costa Rica

e-mail: marietta.flores@ucr.ac.cr

E. Barquero-Calvo

Programa de Investigación en Enfermedades Tropicales, Universidad Nacional de Costa Rica, Heredia, Costa Rica

e-mail: elias.barquero.calvo@una.cr

M. Ramírez

Departamento de Patología Aviar, Escuela de Medicina Veterinaria, Universidad Nacional de Costa Rica, Heredia, Costa Rica

e-mail: marciar228@hotmail.com

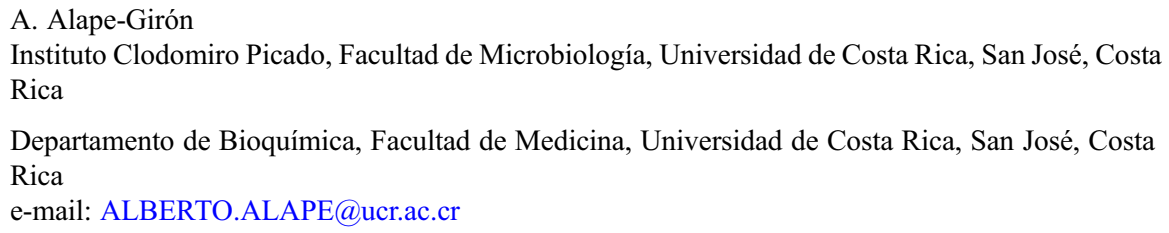

Departamento de Bioquímica, Facultad de Medicina, Universidad de Costa Rica, San José, Costa Rica

e-mail: ALBERTO.ALAPE@ucr.ac.cr 


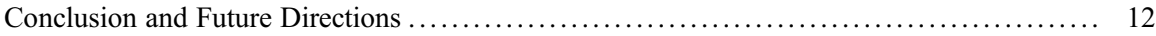

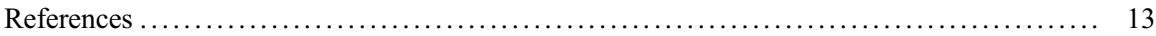

\section{Abstract}

Poultry products represent over $30 \%$ of animal protein consumption worldwide, and its demand is growing considerably. The current global annual production of poultry meat and eggs is more than 115 million tons and 70 million tons, respectively. Necrotic enteritis (NE) in poultry is a reemerging infectious disease caused by certain strains of Clostridium perfringens. This bacterium is found in limited quantities as a normal inhabitant of the birds' gut; however, under certain circumstances, a pathogenic strain proliferates and secretes a variety of bacteriocins, mucins, and adhesins that favors bacteria colonization and establishment. Once bacterial population reaches a certain density, toxin production is triggered which induces mucosal damage. NE generates a dramatic reduction of production levels and a significant increase in mortality in flocks of broilers and laying hens, leading to annual economic losses for the poultry industry estimated to be over $\$ 6$ billion. NE may manifest as an acute or chronic enterotoxemia. Acute infection is associated with higher rates of mortality and chronic infection, with loss of weight and productivity in sick animals. There is evidence that immunization with formalin-inactivated crude supernatants, native or modified toxins, or other proteins induces partial protection against NE. This review summarizes the findings concerning virulence factors associated with NE pathogenesis and the efforts oriented to develop rational strategies to prevent and control this disease.

\section{Keywords}

Bacterial toxins $\bullet$ Bacterial phospholipase $\bullet$ Pore-forming toxin $\bullet$ Necrotic enteritis $\bullet$. perfringens $\bullet$ Enterotoxemia

\section{Introduction}

During the last two decades, the global consumption of poultry meat and eggs has increased considerably, which reflects the dynamics of the poultry industry (FAO 2015, 2016). Poultry meat and eggs are protein-rich products available at relatively low price and therefore are considered basic food in many societies (FAO 2015, 2016). The increasing demands of poultry meat have forced the global poultry industry to improve genetic selection for faster growth, enhance feed conversion, promote higher meat yields, and lower mortality rates (Cooper et al. 2013).

One of the major concerns in poultry meat production is that the birds have healthy intestinal tracts to grow to proper weights. Therefore, the indiscriminate use of antibiotics as growth promoters favoring weight gain of broilers, and helping to control bacterial infections, was allowed for many years (Van Immerseel et al. 2016). However, the risk of antibiotic resistance among human pathogens is favoring the ban of their use as growth promotors around the world, leading to NE reemergence 
in poultry (Van Immerseel et al. 2016). Currently NE is causing annual economic losses for the poultry industry estimated to be over $\$ 6$ billion, on a global scale, due to an impaired growth performance and veterinary treatments (Wade and Keyburn 2015).

$\mathrm{NE}$ in poultry is caused by the overgrowth of pathogenic C. perfringens which induces intestinal mucosal necrosis (Prescott et al. 2016a). However, the presence of a pathogenic $C$. perfringens strain is not sufficient to precipitate this disease, as predisposing factors are necessary to generate the optimal conditions that favor its proliferation (Moore et al. 2016).

C. perfringens is a Gram-positive, anaerobic, spore-forming, mesophilic bacterium widespread in nature and commonly found in the intestines of humans and animals (Uzal et al. 2014). This bacterium produces more than 15 toxins and it is responsible for a variety of diseases in humans, domestic animals, and livestock, even though its ability to cause diseases is strain dependent (Uzal et al. 2014). On poultry farms, C. perfringens can be isolated from soil, birds feces, litter material, live insects, poultry food, water pipes, nipple-drinker drip-cups, floors, wall, fans, and from the clothes and shoes of farm staff (Lee et al. 2011a). C. perfringens spores are very resistant to desiccation, chemicals, and temperature, which causes great risk of infection and reinfection in NE (Uzal et al. 2014). Thus, pathogenic C. perfringens strains could be transmitted by the fecal-oral route as well as through contaminated food, water, housing structures, and insects, as well as by vertical transmission from hens to newly hatched chicks (Lee et al. 2011a).

\section{Necrotic Enteritis: Symptoms, Pathological Findings, and Diagnosis}

NE has been identified in broiler chickens, laying hens, turkeys, ducks, quails, waterfowl, and ostriches in association with certain predisposing factors (Moore et al., 2016). Outbreaks of NE represent the endpoint of a complex series of events, and it occurs either as an acute clinical or as a mild subclinical enterotoxemia (Prescott et al. 2016a).

Birds suffering from clinical NE are depressed, inappetent, huddling, somnolent, diarrheic, dehydrated, and have ruffled feathers (Cooper et al. 2013). The onset of disease is sudden, with birds dying within $1-2 \mathrm{~h}$ after the first appearance of clinical signs, and even without premonitory clinical signs (Cooper et al. 2013). Mortality can reach up to $50 \%$, and productivity losses of up to $1 \%$ per day can last several days (Cooper et al. 2013).

Postmortem findings in clinical cases of NE include the small intestine (usually middle to distal) very relaxed and fragile (Cooper et al. 2013). The duodenum, jejunum, ileum, and cecum are frequently thickened, friable, distended, and filled with gas (Cooper et al. 2013). As the disease progresses, there is a large accumulation of necrotic debris that constitute a dark brown diphtheritic membrane which adheres tightly to the jejunum mucosa and sometimes to the duodenum, ileum, and ceca (Cooper et al. 2013). 
Although clinical outbreaks of NE may cause high levels of mortality, the subclinical form of the disease is more relevant. Sick birds remain untreated because the disease is undetected, generating great economic losses for the poultry industry (Wade and Keyburn 2015).

Subclinical NE occurs without a substantial increase in mortality, but with clear signs of intestinal disorders (Cooper et al. 2013). There is damage of the intestinal mucosa, problems with digestion and absorption, lower performance resulting in weight loss, increased feed conversion rate, and decrease in production (Cooper et al. 2013). Gram-positive rods are found in the lesions and can be isolated by differential anaerobic cultures (Cooper et al. 2013). Necropsy findings of subclinical cases include ulcers of focal necrosis in the mucosal surface, with a watery intestinal content that has a greenish brown color (Cooper et al. 2013). Histologically, there are necrotic foci in the villi of the duodenum and ileum mucosa, accompanied by congestion of blood vessels in the lamina propria and submucosa (Cooper et al. 2013). Often the liver is enlarged and there is cholangiohepatitis or hepatitis. It is proposed that the large number of $C$. perfringens residing in the intestine allows it to get into the bile ducts (Cooper et al. 2013). Livers are distended, have a pale appearance with extensive red or white foci of coagulative necrosis, and contain clusters of Gram-negative bacilli in necrotic areas (Cooper et al. 2013).

Microscopic examination of early stages of NE shows that the lamina propria is hyperaemic and infiltrated with numerous inflammatory cells, mainly granulocytes (Olkowski et al. 2008). There is extensive edema and substantial disturbance of the structural integrity between the lamina propria and the enterocytes (Olkowski et al. 2008). Severe coagulative necrosis of the luminal part of the mucosa is observed in later stages, with the massive presence of Gram-positive bacilli which do not invade the epithelium, nor are they attached to the viable mucosal epithelial cells (Cooper et al. 2013). The presence of a pseudomembrane consisting of numerous Grampositive bacilli suspended in mucus, necrotic epithelial cells, erythrocytes, granulocytes, mononuclear cells, and fibrin occurs in severe cases (Cooper et al. 2013). If the birds survive, the regenerated mucosa epithelium is characterized by a decrease in goblet and columnar epithelial cells and an increase in cuboidal cells, which results in a short flat villus with a reduced absorptive surface (Cooper et al. 2013). In cases of cholangiohepatitis, histopathological lesions include extensive multifocal coagulative necrosis in the liver, bile duct hyperplasia, fibrinoid necrosis, cholangitis, and occasionally focal granulomatous inflammation (Cooper et al. 2013).

Since $C$. perfringens is part of the intestine microbiota of birds, their presence is not indicative of disease. However, an increase up to $10^{9} \mathrm{C}$. perfringens $/ \mathrm{g}$ intestinal content may occur in sick animals, whereas in healthy birds, it increases to not more than $10^{5}$ C. perfringens/g (Cooper et al. 2013). The diagnosis of NE in chickens has been done historically via clinical, macroscopic, and microscopic findings characteristic of the infection (Cooper et al. 2013). A standardized scoring system based on macroscopic lesions is common (Cooper et al. 2013). Currently, new molecular techniques offer the possibility of detecting potentially pathogenic strains in an 
accurate way, for example, identifying genes encoding for toxins relevant for $\mathrm{NE}$ pathogenesis in isolates from clinical samples (Bailey et al. 2013).

\section{Clostridium perfringens Toxins Involved in Necrotic Enteritis}

Although C. perfringens as a species produces more than 15 different toxins, individual strains produce only some of them. Based on four of the toxins produced by $C$. perfringens ( $\alpha, \beta, \varepsilon$, and $\mathrm{i}$ ), strains are classified into five different toxinotypes, from A to E (Uzal et al. 2014). Other toxins, such as perfringolysin O (PFO or $\theta$-toxin), collagenase ( $\kappa$-toxin), $\beta 2$ toxin, CPE, and NetB, are not considered for toxinotyping (Uzal et al. 2014). NE in poultry is caused mainly by $C$. perfringens toxinotype A strains, which among other toxins produce phospholipase $\mathrm{C}$ (CpPLC or $\alpha$ toxin) and the pore-forming necrotic enteritis B-like toxin (NetB) (Uzal et al. 2014).

In C. perfringens, the VirR/VirS two-component regulatory system and the regulatory RNA (VR-RNA) cascade control expression of a regulon composed of 150 genes, including those for some toxins (such as cpa that codifies for CpPLC, $p f o A$, and netb), transporters, and energy metabolism (Ohtani and Shimizu 2015). The accessory growth regulator (Agr)-like quorum-sensing system monitors bacterial environments, regulates, and switches on the VirR/VirS system, and thus toxin production during $\mathrm{NE}$ is greatly influenced by different factors affecting bacterial density (Ohtani and Shimizu 2015).

\section{C. perfringens Phospholipase C (Cp-PLC)}

The cpa gene encoding CpPLC is present on the chromosome of all C. perfringens strains and shows only minor variations of sequence among strains (Uzal et al. 2014). However, the amount of CpPLC produced by different strains varies considerably, reflecting differences in gene expression (Uzal et al. 2014). Toxinotype A strains, which are associated with gas gangrene in humans and with NE in birds, produce the highest amount of CpPLC (Uzal et al. 2014).

The CpPLC is a zinc-dependent metalloenzyme with phosphohydrolase activity and a preference for phosphatidylcholine and sphingomyelin, two main components of the outer layer of the eukaryotic cell membranes (Flores-Díaz et al. 2016). This $43 \mathrm{kDa}$ toxin is composed of an $\mathrm{N}$ - and a C-terminal domain. The catalytically active site is located in the $\mathrm{N}$-terminal domain, while the $\mathrm{C}$-terminal domain is important for anchoring and interaction with biological membranes (Flores-Díaz et al. 2016). CpPLC possibly anchors to membranes via $\mathrm{Ca}^{2+}$ ion binding sites in the $\mathrm{C}$-terminal domain, and thus the active site is oriented to interact directly with membrane phospholipids (Flores-Díaz et al. 2016).

It has been shown that CpPLC affects the jejunal mucosa of laying hens, which suggests that it contributes to NE pathogenesis (Rehman et al. 2006). However, CpPLC is not the main virulence factor in this disease, as it has been shown that a 
pathogenic $C$. perfringens strain with inactivated cpa gene still induces NE lesions comparable to those found in birds challenged with the wild-type strain (Rood et al. 2016).

\section{C. perfringens NetB}

Virulent $C$. perfringens strains associated with NE secrete into culture supernatant a pore-forming toxin, referred to as NetB, which has $30 \%$ sequence similarity with S. aureus alpha toxin (Rood et al. 2016). Treatment of chicken cells with NetB causes rapid cell blebbing and swelling, leading to cell lysis. To determine the role of NetB in NE, a mutant strain isolated from sick chickens lacking the netb gene was generated by homologous recombination (Rood et al. 2016). That mutant strain does not induce NE, while the wild-type strain and the mutant strain complemented with the wild type netb gene did, demonstrating that NetB is essential for causing experimental NE in chickens (Rood et al. 2016). More recent work has shown that the coding sequence of the netb gene is highly conserved and that strains producing the most severe injuries are those that produce greater amounts of NetB (Rood et al. 2016).

The crystal structure of monomeric NetB shows an overall $\beta$-rich fold similar to those of the alpha-hemolysin family, such as the delta toxin from $C$. perfringens, S. aureus Panton-Valentine leukocidin S, S. aureus leukotoxin LukE, and S. aureus alpha-hemolysin (Yan et al. 2013). NetB consists of $16 \beta$-strands and an $\alpha$-helix, arranged into a $\beta$-sandwich, latch, rim, and prestem domains (Yan et al. 2013). The $\beta$-sandwich domain is comprised of a five-stranded and six-stranded antiparallel $\beta$-sheets (Yan et al. 2013). The prestem region (residues 140-186) forms a threestranded antiparallel $\beta$-sheet, which packs against the five-stranded antiparallel $\beta$-sheet of the $\beta$-sandwich (Yan et al. 2013). The rim region, which is involved in membrane recognition and binding, is comprised of a four-stranded antiparallel $\beta$-sheet and a well-ordered loop formed by residues 205 to 242 (Yan et al. 2013). The base of the rim domain contains a number of solvent-exposed aromatic groups predicted to form direct contacts with the lipid membrane. Structural comparisons with other members of the alpha-hemolysin family reveal significant differences in the conformation of the membrane binding domain, suggesting that NetB may recognize different membrane receptors or use a different mechanism for membrane-protein interactions (Yan et al. 2013). Entrance from either side of the NetB pore is lined with acidic residues (Asp-116, Glu-153, and Glu-132) forming two distinct negatively charged belts, which likely explains why the NetB pores differ in their ion selectivity, preferring cations over anions (Yan et al. 2013).

The crystal structure of the heptameric complex of NetB in detergent, which likely represents the membrane-inserted pore form, is known (Savva et al. 2013). A NetB oligomer is composed of seven monomers arranged in a ring, in which each monomer forms an extensive hydrogen bond network and salt bridges on both interfaces (Savva et al. 2013). The seven $\beta$-sandwich domains form the cap of the mushroom-shaped heptamer, whereas the stem domains form the 14-strand $\beta$-barrel 
pore (Savva et al. 2013). This heptameric assembly shares structural similarity to that of the staphylococcal $\alpha$-hemolysin, although important differences can be identified in the membrane binding and pore lumen regions (Savva et al. 2013).

Site-directed mutagenesis studies helped identify several residues critical for NetB-induced cell lysis, which are clustered in regions predicted to be required for oligomerization or membrane binding (Yan et al. 2013). Comparative analysis of the NetB structure indicates that the S254 residue is involved in oligomerization, as a NetB S254 L variant does not oligomerize in lipid bilayers and chicken erythrocytes (Yan et al. 2013). In contrast, the NetB R230Q and W287R variants form oligomers in a solution and also form single channels in artificial phospholipid bilayers; however, these protein variants were inactive against chicken erythrocytes (Yan et al. 2013). Since the R230 and W287 residues are located in the putative rim domain of NetB, it was postulated that these residues are required for binding to a cell-surface receptor (Yan et al. 2013).

\section{C. perfringens TpeL}

A large clostridial glucosylating cytotoxin referred to as TpeL, and homologous to TcdA and TcdB from $C$. difficile, also appears to have a role in the pathogenesis of NE (Chen and McClane 2015). TpeL is encoded on a conjugative plasmid (Chen and McClane 2015) and binds to LDL receptor-related protein 1 in the target cells (Schorch et al. 2014). Experimental infections with $C$. perfringens TpeL-positive strains in broilers induce more severe intestinal lesions and cause NE, with a more rapid course and higher fatality rate than those strains lacking this cytotoxin (Coursodon et al. 2012). Thus, TpeL likely potentiates the effect of other C. perfringens virulence factors associated with NE.

\section{Other Toxins}

A chromosomally encoded bacteriocin referred to as perfrin, present in several NetB positive strains of broiler origin, likely contributes to the virulence of $\mathrm{NE}$ associated C. perfringens strains (Timbermont et al. 2014). Perfrin has bactericidal activity over a wide $\mathrm{pH}$-range and has no sequence homology to any currently known bacteriocin, suggesting that this is the paradigm for a new bacteriocin class (Timbermont et al. 2014). Perfrin inhibits the growth of commensal $C$. perfringens strains and seemingly confers an advantage to pathogenic strains during competition for nutrients (Timbermont et al. 2009).

Comprehensive studies of $C$. perfringens genomes from several NE-inducing strains have revealed up to four different, large conjugative plasmids encoding several known and putative virulence factors clustered in discrete pathogenicity loci (Lepp et al. 2010; Parreira et al. 2012; Lepp et al. 2013). The best characterized of them, referred to as NELoc-1, encodes the netb gene and 36 additional genes including two encoding glycohydrolases, one metalloprotease, two leukocidins, one 
protein similar to internalin, and several putative adhesins (Lepp et al. 2010). Thus, besides the well-recognized toxins, NE pathogenesis likely involves several additional enzymes and adhesins encoded in those pathogenicity loci, and hence, variation in plasmid contents probably contributes greatly to interstrain pathogenicity variation (Lacey et al. 2016).

\section{Predisposing Factors}

Besides the presence of a $C$. perfringens virulent strain in the intestine, certain predisposing factors that affect gut microbiota or the immune state of the birds are required for the onset of NE (Moore 2016).

One of the most important predisposing factors to NE is the intestinal mucosa damage caused by the intracellular phase of coccidial lifecycle (Wu et al. 2014; Moore 2016; Tsiouris 2016). Coccidiosis induces extravasation of plasma proteins into the intestinal lumen which favors the overgrowth of pathogenic C. perfringens (Moore 2016). Furthermore, coccidial infections enhance intestinal mucus production which also creates a favorable environment for $C$. perfringens proliferation and toxin production (Moore 2016).

Feed composition is another important factor that could favor the onset of NE (Moore 2016; Tsiouris 2016). High content of proteins from animal sources is a dietary factor that predisposes broilers to NE (Wu et al. 2014). Diets with high viscosity, due to a high content of indigestible materials soluble in water, predispose the bird to intestinal stasis which enables proliferation of $C$. perfringens (Moran 2014). The incidence of NE in animals fed with diets rich in rye, wheat, and barley is 6 to 10 times greater than in those fed with diets based on maize (Moore 2016).

Stress in broiler chickens, such as that induced by high stocking density, predisposes the birds to NE in a subclinical experimental model (Tsiouris et al. 2015). Immunosuppressive agents such as the viruses that cause chick anemia, Gumboro disease or Marek's disease, or exposure to mycotoxins, reduce resistance to gut infections and may increase the severity of NE (Antonissen et al. 2014, 2015; Moore 2016).

\section{Experimental Models of the Disease}

NE produced by $C$. perfringens is a complex and multifactorial disease difficult to reproduce experimentally (Shojadoost et al. 2012; Prescott et al. 2016b; Van Waeyenberghe et al. 2016). Several factors should be considered for a successful reproduction of experimental $\mathrm{NE}$, including induction of intestinal damage with a coccidial infection, genetic background of the animals, size of feed particles, animal protein content and non-starch polysaccharides in the birds'diet, and C. perfringens strains used in the challenge, as well as the route, dose, and frequency of the infection (Stanley et al. 2014; Rodgers et al. 2015; Prescott et al. 2016b; Van Waeyenberghe et al. 2016; Oh and Lillehoj 2016). 
To predispose for experimental NE, coccidial vaccines are often administered at 10 times the recommended vaccination dose, 4-5 days before the $C$. perfringens challenge, so that the coccidian-induced intestinal damage coincides with bacterial challenge (Stanley et al. 2014; Rodgers et al. 2015; Prescott et al. 2016b; Van Waeyenberghe et al. 2016).

There are different susceptibilities of Cobb, Ross, and Hubbard broilers toward developing experimental NE. When chickens are orally infected with E. maxima and viable $C$. perfringens and fed a high-protein diet to promote the development of experimental NE; Cobb chickens are more susceptible to NE than the Ross and Hubbard lines (Jang et al. 2013).

Diets that increase the digesta viscosity or enhance mucus production lead to prolonged transit times in the intestine, favoring C. perfringens growth (Prescott et al. 2016b). High ground feed; cereals that contain high percentages of watersoluble, indigestible non-starch polysaccharide; and diets with large amounts of animal protein, such as fish meal, allow bacteria to proliferate faster and predispose birds to experimental NE (Stanley et al. 2014; Rodgers et al. 2015; Prescott et al. 2016b).

The use of netb-positive $C$. perfringens strains with other virulence determinants, such as the toxin tpeL, is critical for reproducing NE experimentally. The plasmids associated to virulence factors can be lost on subcultures of the bacterium; therefore, maintaining a frozen master stock of the virulent strains, and confirming by PCR that the strain used for challenge has not lost netb carrying plasmid, is mandatory (Prescott et al. 2016b).

Fluid thioglycolate medium (with dextrose) is the most common culture medium used as the challenge inoculum. To induce NE experimentally, birds are usually starved overnight and fed with infected food, inoculated at a ratio of 1.25-1.5 fluid thioglycolate medium/feed (v/w), twice daily (Prescott et al. 2016b). Cultures of $15 \mathrm{~h}$ increase lesion scores, probably due to greater toxin production (Prescott et al. 2016b).

\section{Pathogenesis}

NE pathogenesis depends on the concerted expression of a variety of extracellular enzymes and toxins by $C$. perfringens which contributes to the colonization of the intestinal mucosa, acquisition of nutrients, evasion of host defenses, and the damage to host tissues (Parreira et al. 2016; Prescott et al. 2016a).

A higher number of pathogenic $C$. perfringens strains produce bacteriocins compared with commensal $C$. perfringens strains (Timbermont et al. 2014). By inhibiting other $C$. perfringens strains, the virulent strains obtain the maximum benefit of the increased nutrient availability due to the initial damage of the intestinal mucosa (Timbermont et al. 2014). Mucus secreted by intestinal epithelial cells is rich in glycosylated mucin glycoproteins and contains a variety of antimicrobial molecules designed to add a barrier to bacterial colonization of the intestinal epithelial cells (Prescott et al. 2016a). Strains of $C$. perfringens associated to NE possess 
within NELoc-1 two genes encoding "chitinases" capable of degrading mucins (Prescott et al. 2016a). These enzymes probably hydrolyze the chitobiose core of glycoproteins present in the intestinal mucus and therefore participate in C. perfringens colonization (Prescott et al. 2016a). The sugars of glycoproteins and glycolipids represent potential binding sites for microbial adhesins, as well as an energy source (Prescott et al. 2016a). C. perfringens sialidases, which are regulated by the VirR/VirS system, could be involved in promoting bacterial adhesion through modification of epithelial surfaces which expose receptors on enterocyte cell membranes ( $\mathrm{Li}$ and McClane 2014). The adhesion of C. perfringens to extracellular matrix-based collagen type III and types IV and V, fibrinogen, and vitronectin depends on a putative fimbrial adhesins VR-10B operon, always present in $n e t B$-positive isolates (Lepp et al. 2013). This operon is important for colonization because its inactivation abolishes collagen adhesion, as well as virulence (Wade et al. 2015).

Virulent $C$. perfringens strains either secrete collagenases at higher levels than commensal strains or induce the expression of host collagenolytic enzymes because higher collagenase activity is found in the intestines of broilers with NE than in controls (Olkowski et al. 2008). Ultrastructural analysis of NE found that primary changes occur at the level of basal and lateral domains of the enterocytes, whereas their apical domain remains intact even in the face of advanced necrotic changes (Olkowski et al. 2008). Thus, mucosal necrosis seems to result from the destruction of lamina propria, the extracellular matrix, and intercellular junctions (Olkowski et al. 2008). NetB may induce necrotic lesions by forming pores in enterocytes leading to cell death (Rood et al. 2016). Whether this is involved in the early, or later, stages of disease progression is not clear (Prescott et al. 2016a).

\section{Prevention and Control}

The strategy most commonly used in the control of NE in poultry includes administration of antibiotics (Caly et al. 2015). However, the European ban on antibiotics (feed additives regulation 1831/2003/EC), which took effect in January 2006, made necessary other alternatives to prevent NE occurrence (Caly et al. 2015). NE prophylactic treatments include improving the hygiene in poultry farms, coccidiosis prevention, diet modifications to include prebiotics and probiotics, or targeting directly C. perfringens virulent strains with bacteriophages (Caly et al. 2015).

Prebiotics are mostly indigestible oligosaccharides that will stimulate the commensal microbiota and enhance the beneficial effects of probiotics within the host, dependent upon dose, length of treatment, or even diet (Caly et al. 2015). Mannanoligosaccharides and Saf-Mannan are components within the yeast cell wall often used as feed additives not only to improve intestinal health and immune response but also inhibit pathogen colonization by reducing adhesion (Thanissery et al. 2010; Caly et al. 2015).

Probiotics are a live microbial food supplement that improve immunity and increase broiler chicken performance (health, weight gain, feed conversion), thus 
reducing the risk of infection by opportunistic pathogens in the host (Caly et al. 2015). It has been shown that enterococci, lactic acid bacteria, and several Bacillus species (licheniformis, pumilus, subtilis, cereus) have antagonistic activity against C. perfringens, associated with the production of bacteriocins and antimicrobial peptides (Knap et al. 2010; Cao et al. 2012; Jayaraman et al. 2013; Caly et al. 2015).

Bacteriophage therapy was widely used to treat bacterial infections up until the $40 \mathrm{~s}$ and has had a recent upsurge of interest with the growing need for alternatives to antibiotic treatments (Caly et al. 2015). Bacteriophages produce endolysins, which target peptidoglycans and lyse the bacterial cell wall, freeing the phages and allowing them to spread to other cells (Caly et al. 2015). Many bacteriophages isolated from C. perfringens strains of poultry origin, with specific anti-Clostridium activity, have been described and used to limit $C$. perfringens infection in field trials (Miller et al. 2010). A mixture of six bacteriophages reduces mortality in a NE challenge by over $90 \%$, and overall performance was enhanced as assessed by weight gain and feed conversion (Miller et al. 2010).

\section{Immunity and Prospects for Vaccination}

The immune response to $C$. perfringens infection in poultry is not completely understood (Mot et al. 2014). Several C. perfringens antigens are recognized by antibodies present in sera from chickens after a NE infection, including pyruvate: ferredoxin oxidoreductase (PFOR), elongation factor G, perfringolysin O, glyceraldehyde-3-phosphate dehydrogenase (GAPDH), fructose-1,6-biphosphate aldolase (FBA), endo-beta-N-acetylglucosaminidase (Naglu) and phosphoglyceromutase (Pgm), the elongation factor $\mathrm{Tu}$, and a hypothetical unidentified protein (HP) (Kulkarni et al. 2006; Lee et al. 2011a, b).

Many trials with different approaches have been carried out to develop a vaccine able to induce a protective immunity against NE (Mot et al. 2014). The antigens have been administered as non-inactivated supernatants, formalin-inactivated crude toxoids, immunogenic proteins, and genetically modified toxins, using different routes of administration (intramuscularly, subcutaneously, orally), varying dosages, several boosters, and adjuvants. Furthermore, selected C. perfringens antigens have also been delivered by live-attenuated vaccine carrier strains. Multiple vaccination dosages are necessary for a good immune response, and one parenteral single vaccination, at the day of hatching, offers no protection (Jiang et al. 2009; Crouch et al. 2010; Lanckriet et al. 2010; Kulkarni et al. 2010; Saleh et al. 2011; Mot et al. 2013; Keyburn et al. 2013a, b; Jiang et al. 2015).

Chickens immunized intramuscularly two (or three) times with recombinant CpPLC/CpPLC toxoid, GAPDH, HP, FBA, or PFOR, were able to decrease the mean intestinal lesion score (Kulkarni et al. 2007). GAPDH and FBA protected only against mild challenge, whereas CpPLC, HP, and PFOR protected significantly against a heavy challenge (Kulkarni et al. 2007). In another study, double subcutaneous vaccination regimens using CpPLC, NetB toxin, PFOR, or elongation factor $\mathrm{Tu}$ gave similar protection levels after experimental infection (Jang et al. 2012). 
Immunization with Naglu and Pgm also confers partial protection after challenge with two different strains (Jiang et al. 2009).

$\mathrm{CpPLC}$ has been considered a potential antigen for a vaccine to protect from NE in poultry (Kulkarni et al. 2006). Even though CpPLC has been shown not to be crucial for the pathogenesis of $\mathrm{NE}$, immunization with this toxin confers at least partial protection against NE (Kulkarni et al. 2006; Zekarias et al. 2008). It has been postulated that secreted proteins of Gram-positive bacteria accumulate within the cytoplasm and can be recognized by antibodies that might block protein transport channels, thereby inhibiting bacterial proliferation (Zekarias et al. 2008). Different trials have shown that immunization of birds with CpPLC, or its carboxy terminal domain only, either intramuscularly or with Salmonella strains expressing those antigens, are partially protected against the mucosal damage induced experimentally by virulent $C$. perfringens strains (Kulkarni et al. 2006; Zekarias et al. 2008; Hoang et al. 2008; Cooper et al. 2009; Kulkarni et al. 2010; Jang et al. 2012, 2015; Fernandes da Costa et al. 2016).

Comparison of different vaccination schedules, different challenge models, and different scoring procedures to induce protection against $\mathrm{NE}$ has revealed that vaccination with NetB (using recombinant NetB, native NetB, or toxoids and bacterin vaccines containing NetB) induces an immune response that partially protects against NE. These results occur in directly vaccinated birds or in chicks derived from vaccinated hens (Jang et al. 2012; Keyburn et al. 2013a, b; Fernandes da Costa et al. 2013; Jiang et al. 2015; Fernandes da Costa et al. 2016). Recently, a novel attenuated Salmonella vaccine strain composed of the carboxyl-terminal fragment of CpPLC and a GST-netB fusion protein designed to replicate 6-10 times before lysis in broilers was able to generate a strong production of intestinal IgA, IgY, and IgM antibodies specific against those toxins that protect against mild and severe challenges of pathogenic $C$. perfringens (Jiang et al. 2015). All of the above-described data thus shows that the development of a practical and fully protective vaccine against NE is a difficult challenge and requires the combination of at least two different bacterial proteins.

\section{Conclusion and Future Directions}

The annual consumption of poultry eggs and meat for the global human population is respectively more than 70 million and 115 million tons, and the demand is growing. Therefore, an efficient and safe production of healthy poultry is essential for the future. The indiscriminate use of antibiotics as growth promoters favoring weight gain of poultry and helping to control bacterial infections has been banned in several countries, leading to NE reemergence and entailing enormous economic losses worldwide. Because there is a rising concern that the antibiotic-resistant pathogens could be transmitted to humans, it is expected that the use of antimicrobials in animal production will decrease during the coming years.

To ensure continuity in the supply of poultry-based food products, effective control measures against NE in the framework of the current situation are required. 
Under optimal conditions, pathogenic $C$. perfringens strains proliferate in birds' gut causing intestinal mucosal necrosis, diarrhea, malabsorption, dehydration, and, in severe cases, death. Several virulence factors, such as chitinases, bacteriocins, and toxins, likely play a role in colonization and intestinal mucosal damage induced by the bacteria. The new possibilities provided by genomic and proteomic techniques offer alternatives to achieve a comprehensive knowledge of the pathogenesis of NE. A better understanding of the complex interaction between pathogenic C. perfringens and nonpathogenic commensal bacteria in the birds' gut is also required to implement rational strategies to control NE. Further studies on the immunobiology of host-pathogen interaction will contribute toward identifying factors that determine the occurrence of NE outbreaks, allowing the development of integrated and effective strategies to affordably prevent and treat this disease.

Acknowledgments This work was supported by CONICIT (FI-034B-14), FEES CONARE, and Vicerrectoría de Investigación Universidad de Costa Rica (grants 741-B5-504 and 741-B5-656).

\section{References}

Antonissen G, Van Immerseel F, Pasmans F, Ducatelle R, Haesebrouck F, Timbermont L, Verlinden M, Janssens GP, Eeckhaut V, Eeckhout M, De Saeger S, Hessenberger S, Martel A, Croubels S. The mycotoxin deoxynivalenol predisposes for the development of Clostridium perfringens-induced necrotic enteritis in broiler chickens. PLoS One. 2014;9:e108775.

Antonissen G, Croubels S, Pasmans F, Ducatelle R, Eeckhaut V, Devreese M, Verlinden M, Haesebrouck F, Eeckhout M, De Saeger S, Antlinger B, Novak B, Martel A, Van Immerseel F. Fumonisins affect the intestinal microbial homeostasis in broiler chickens, predisposing to necrotic enteritis. Vet Res. 2015;46:98.

Bailey MA, Macklin KS, Krehling JT. Use of a multiplex PCR for the detection of toxin-encoding genes netB and tpeL in strains of Clostridium perfringens. ISRN Vet Sci. 2013;2013:865702.

Caly DL, D'Inca R, Auclair E, Drider D. Alternatives to antibiotics to prevent necrotic enteritis in broiler chickens: a microbiologist's perspective. Front Microbiol. 2015;6:1336.

Cao L, Yang XJ, Li ZJ, Sun FF, Wu XH, Yao JH. Reduced lesions in chickens with Clostridium perfringens-induced necrotic enteritis by Lactobacillus fermentum 1.20291. Poult Sci. 2012;91:3065-71.

Chen J, McClane BA. Characterization of Clostridium perfringens TpeL toxin gene carriage, production, cytotoxic contributions, and trypsin sensitivity. Infect Immun. 2015;83:2369-81.

Cooper KK, Trinh HT, Songer JG. Immunization with recombinant alpha toxin partially protects broiler chicks against experimental challenge with Clostridium perfringens. Vet Microbiol. 2009;133:92-7.

Cooper KK, Songer JG, Uzal FA. Diagnosing clostridial enteric disease in poultry. J Vet Diagn Invest. 2013;25:314-27.

Coursodon CF, Glock RD, Moore KL, Cooper KK, Songer JG. TpeL-producing strains of Clostridium perfringens type A are highly virulent for broiler chicks. Anaerobe. 2012;18:117-21.

Crouch CF, Withanage GS, de Haas V, Etore F, Francis MJ. Safety and efficacy of a maternal vaccine for the passive protection of broiler chicks against necrotic enteritis. Avian Pathol. 2010;39:489-97.

FAO. Food outlook: biannual report on global markets. 2016. ISSN: 0251-1959

FAO. World agriculture: towards 2015/2030. 2015. ISBN: 9251048355 
Fernandes da Costa SP, Mot D, Bokori-Brown M, Savva CG, Basak AK, Van Immerseel F, Titball RW. Protection against avian necrotic enteritis after immunisation with NetB genetic or formaldehyde toxoids. Vaccine. 2013;31(37):4003-8.

Fernandes da Costa SP, Mot D, Geeraerts S, Bokori-Brown M, Van Immerseel F, Titball RW. Variable protection against experimental broiler necrotic enteritis after immunization with the C-terminal fragment of Clostridium perfringens alpha-toxin and a non-toxic NetB variant. Avian Pathol. 2016;45:381-8.

Flores-Díaz M, Monturiol-Gross L, Naylor C, Alape-Girón A, Flieger A. Bacterial sphingomyelinases and phospholipases as virulence factors. Microbiol Mol Biol Rev. 2016;80 (3):597-628.

Hoang TH, Hong HA, Clark GC, Titball RW, Cutting SM. Recombinant Bacillus subtilis expressing the Clostridium perfringens alpha toxoid is a candidate orally delivered vaccine against necrotic enteritis. Infect Immun. 2008;76:5257-65.

Jang SI, Lillehoj HS, Lee SH, Lee KW, Lillehoj EP, Hong YH, An DJ, Jeong W, Chun JE, Bertrand F, Dupuis L, Deville S, Arous JB. Vaccination with Clostridium perfringens recombinant proteins in combination with Montanide ${ }^{\mathrm{TM}}$ ISA 71 VG adjuvant increases protection against experimental necrotic enteritis in commercial broiler chickens. Vaccine. 2012;30:5401-6.

Jang SI, Lillehoj HS, Lee SH, Lee KW, Lillehoj EP, Hong YH, An DJ, Jeoung DH, Chun JE. Relative disease susceptibility and clostridial toxin antibody responses in three commercial broiler lines coinfected with Clostridium perfringens and Eimeria maxima using an experimental model of necrotic enteritis. Avian Dis. 2013;57:684-7.

Jayaraman S, Thangavel G, Kurian H, Mani R, Mukkalil R, Chirakkal H. Bacillus subtilis PB6 improves intestinal health of broiler chickens challenged with Clostridium perfringens-induced necrotic enteritis. Poult Sci. 2013;92:370-4.

Jiang Y, Kulkarni RR, Parreira VR, Prescott JF. Immunization of broiler chickens against Clostridium perfringens-induced necrotic enteritis using purified recombinant immunogenic proteins. Avian Dis. 2009;53:409-15.

Jiang Y, Mo H, Willingham C, Wang S, Park JY, Kong W, Roland KL, Curtiss 3rd R. Protection against necrotic enteritis in broiler chickens by regulated delayed lysis salmonella vaccines. Avian Dis. 2015;59:475-85.

Keyburn AL, Portela RW, Ford ME, Bannam TL, Yan XX, Rood JI, Moore RJ. Maternal immunization with vaccines containing recombinant NetB toxin partially protects progeny chickens from necrotic enteritis. Vet Res. 2013a;44:108.

Keyburn AL, Portela RW, Sproat K, Ford ME, Bannam TL, Yan X, Rood JI, Moore RJ. Vaccination with recombinant NetB toxin partially protects broiler chickens from necrotic enteritis. Vet Res. 2013b;44:54.

Knap I, Lund B, Kehlet AB, Hofacre C, Mathis G. Bacillus licheniformis prevents necrotic enteritis in broiler chickens. Avian Dis. 2010;54:931-5.

Kulkarni RR, Parreira VR, Sharif S, Prescott JF. Clostridium perfringens antigens recognized by broiler chickens immune to necrotic enteritis. Clin Vaccine Immunol. 2006;13:1358-62.

Kulkarni RR, Parreira VR, Sharif S, Prescott JF. Immunization of broiler chickens against Clostridium perfringens-induced necrotic enteritis. Clin Vaccine Immunol. 2007;14:1070-7.

Kulkarni RR, Parreira VR, Jiang YF, Prescott JF. A live oral recombinant Salmonella enterica serovar typhimurium vaccine expressing Clostridium perfringens antigens confers protection against necrotic enteritis in broiler chickens. Clin Vaccine Immunol. 2010;17:205-14.

Lacey JA, Johanesen PA, Lyras D, Moore RJ. Genomic diversity of necrotic enteritis-associated strains of Clostridium perfringens: a review. Avian Pathol. 2016;45:302-7.

Lanckriet A, Timbermont L, Eeckhaut V, Haesebrouck F, Ducatelle R, Van Immerseel F. Variable protection after vaccination of broiler chickens against necrotic enteritis using supernatants of different Clostridium perfringens strains. Vaccine. 2010;28:5920-3. 
Lee KW, Lillehoj HS, Jeong W, Jeoung HY, An DJ. Avian necrotic enteritis: experimental models, host immunity, pathogenesis, risk factors, and vaccine development. Poult Sci. 2011a;90:1381-90.

Lee K, Lillehoj HS, Li G, Park MS, Jang SI, Jeong W, Jeoung HY, An DJ, Lillehoj EP. Identification and cloning of two immunogenic Clostridium perfringens proteins, elongation factor $\mathrm{Tu}$ (EF-Tu) and pyruvate: ferredoxin oxidoreductase (PFO) of C. perfringens. Res Vet Sci. 2011b;91:e80-6.

Lepp D, Roxas B, Parreira VR, Marri PR, Rosey EL, Gong J, Songer JG, Vedantam G, Prescott JF. Identification of novel pathogenicity loci in Clostridium perfringens strains that cause avian necrotic enteritis. PLoS One. 2010;5:e10795.

Lepp D, Gong J, Songer JG, Boerlin P, Parreira VR, Prescott JF. Identification of accessory genome regions in poultry Clostridium perfringens isolates carrying the netB plasmid. $\mathrm{J}$ Bacteriol. 2013;195:1152-66.

Li J, McClane BA. Contributions of NanI sialidase to Caco-2 cell adherence by Clostridium perfringens type $\mathrm{A}$ and $\mathrm{C}$ strains causing human intestinal disease. Infect Immun. 2014;82:4620-30.

Miller RW, Skinner EJ, Sulakvelidze A, Mathis GF, Hofacre CL. Bacteriophage therapy for control of necrotic enteritis of broiler chickens experimentally infected with Clostridium perfringens. Avian Dis. 2010;54:33-40.

Moore RJ. Necrotic enteritis predisposing factors in broiler chickens. Avian Pathol. 2016;45:275-81.

Moran Jr ET. Intestinal events and nutritional dynamics predispose Clostridium perfringens virulence in broilers. Poult Sci. 2014;93:3028-36.

Mot D, Timbermont L, Delezie E, Haesebrouck F, Ducatelle R, Van Immerseel F. Day-of-hatch vaccination is not protective against necrotic enteritis in broiler chickens. Avian Pathol. 2013;42:179-84.

Mot D, Timbermont L, Haesebrouck F, Ducatelle R, Van Immerseel F. Progress and problems in vaccination against necrotic enteritis in broiler chickens. Avian Pathol. 2014;43:290-300.

Oh ST, Lillehoj HS. The role of host genetic factors and host immunity in necrotic enteritis. Avian Pathol. 2016;45:313-6.

Ohtani K, Shimizu T. Regulation of toxin gene expression in Clostridium perfringens. Res Microbiol. 2015;166:280-9.

Olkowski AA, Wojnarowicz C, Chirino-Trejo M, Laarveld B, Sawicki G. Sub-clinical necrotic enteritis in broiler chickens: novel etiological consideration based on ultra-structural and molecular changes in the intestinal tissue. Res Vet Sci. 2008;85:543-53.

Parreira VR, Costa M, Eikmeyer F, Blom J, Prescott JF. Sequence of two plasmids from Clostridium perfringens chicken necrotic enteritis isolates and comparison with $C$. perfringens conjugative plasmids. PLoS One. 2012;7:e49753.

Parreira VR, Russell K, Athanasiadou S, Prescott JF. Comparative transcriptome analysis by RNAseq of necrotic enteritis Clostridium perfringens during in vivo colonization and in vitro conditions. BMC Microbiol. 2016;16(1):186.

Prescott JF, Parreira VR, Mehdizadeh Gohari I, Lepp D, Gong J. The pathogenesis of necrotic enteritis in chickens: what we know and what we need to know: a review. Avian Pathol. 2016a;45(3):288-94.

Prescott JF, Smyth JA, Shojadoost B, Vince A. Experimental reproduction of necrotic enteritis in chickens: a review. Avian Pathol. 2016b;45(3):317-22.

Rehman H, Awad WA, Lindner I, Hess M, Zentek J. Clostridium perfringens alpha toxin affects electrophysiological properties of isolated jejunal mucosa of laying hens. Poult Sci. 2006;85:1298-302.

Rodgers NJ, Swick RA, Geier MS, Moore RJ, Choct M, Wu SB. A Multifactorial analysis of the extent to which eimeria and fishmeal predispose broiler chickens to necrotic enteritis. Avian Dis. 2015;59:38-45. 
Rood JI, Keyburn AL, Moore RJ. NetB and necrotic enteritis: the hole movable story. Avian Pathol. 2016;45:295-301.

Saleh N, Fathalla SI, Nabil R, Mosaad AA. Clinicopathological and immunological studies on toxoids vaccine as a successful alternative in controlling clostridial infection in broilers. Anaerobe. 2011;17(6):426-30.

Savva CG, Fernandes da Costa SP, Bokori-Brown M, Naylor CE, Cole AR, Moss DS, Titball RW, Basak AK. Molecular architecture and functional analysis of NetB, a pore-forming toxin from Clostridium perfringens. J Biol Chem. 2013;288:3512-22.

Schorch B, Song S, van Diemen FR, Bock HH, May P, Herz J, Brummelkamp TR, Papatheodorou P, Aktories K. LRP1 is a receptor for Clostridium perfringens TpeL toxin indicating a two-receptor model of clostridial glycosylating toxins. Proc Natl Acad Sci U S A. 2014;111(17):6431-6.

Shojadoost B, Vince AR, Prescott JF. The successful experimental induction of necrotic enteritis in chickens by Clostridium perfringens: a critical review. Vet Res. 2012;43:74.

Stanley D, Wu SB, Rodgers N, Swick RA, Moore RJ. Differential responses of cecal microbiota to fishmeal, Eimeria and Clostridium perfringens in a necrotic enteritis challenge model in chickens. PLoS One. 2014;9:e104739.

Thanissery R, McReynolds JL, Conner DE, Macklin KS, Curtis PA, Fasina YO. Evaluation of the efficacy of yeast extract in reducing intestinal Clostridium perfringens levels in broiler chickens. Poult Sci. 2010;89(11):2380-8.

Timbermont L, De Smet L, Van Nieuwerburgh F, Parreira VR, Van Driessche G, Haesebrouck F, Ducatelle R, Prescott J, Deforce D, Devreese B, Van Immerseel F. Perfrin, a novel bacteriocin associated with netB positive Clostridium perfringens strains from broilers with necrotic enteritis. Vet Res. 2014;45:40.

Tsiouris V, Georgopoulou I, Batzios C, Pappaioannou N, Ducatelle R, Fortomaris P. High stocking density as a predisposing factor for necrotic enteritis in broiler chicks. Avian Pathol. 2015;44:59-66.

Tsiouris V. Poultry management: a useful tool for the control of necrotic enteritis in poultry. Avian Pathol. 2016;45:323-5.

Uzal FA, Freedman JC, Shrestha A, Theoret JR, Garcia J, Awad MM, Adams V, Moore RJ, Rood JI, McClane BA. Towards an understanding of the role of Clostridium perfringens toxins in human and animal disease. Future Microbiol. 2014;9:361-77.

Van Immerseel F, Lyhs U, Pedersen K, Prescott JF. Recent breakthroughs have unveiled the many knowledge gaps in Clostridium perfringens-associated necrotic enteritis in chickens: the first international conference on necrotic enteritis in poultry. Avian Pathol. 2016;45:269-70.

Van Waeyenberghe L, De Gussem M, Verbeke J, Dewaele I, De Gussem J. Timing of predisposing factors is important in necrotic enteritis models. Avian Pathol. 2016;45:370-5.

Wade B, Keyburn AL. The true cost of necrotic enteritis. World Poult. 2015;31:16-7.

Wade B, Keyburn AL, Seemann T, Rood JI, Moore RJ. Binding of Clostridium perfringens to collagen correlates with the ability to cause necrotic enteritis in chickens. Vet Microbiol. 2015;180:299-303.

Wu SB, Rodgers N, Swick RA, Moore RJ. Differential responses of cecal microbiota to fishmeal, Eimeria and Clostridium perfringens in a necrotic enteritis challenge model in chickens. PLoS One. 2014;9:e104739.

Yan XX, Porter CJ, Hardy SP, Steer D, Smith AI, Quinsey NS, Hughes V, Cheung JK, Keyburn AL, Kaldhusdal M, Moore RJ, Bannam TL, Whisstock JC, Rood JI. Structural and functional analysis of the pore-forming toxin NetB from Clostridium perfringens. MBio. 2013;4: e00019-3.

Zekarias B, Mo H, Curtiss 3rd R. Recombinant attenuated Salmonella enterica serovar typhimurium expressing the carboxy-terminal domain of alpha toxin from Clostridium perfringens induces protective responses against necrotic enteritis in chickens. Clin Vaccine Immunol. 2008;15:805-16. 